\title{
Unusual clinical picture of food-dependent exercise-induced anaphylaxis associated with soybean allergy and a reverse sequence of causative factors
}

\author{
Anna Radlińska, Krzysztof Gomułka, Anna Zaleska, Wojciech Mędrala \\ Department of Internal Medicine, Pneumonology and Allergology, Wroclaw Medical University, Wroclaw, Poland \\ Adv Dermatol Allergol 2021; XXXVIII (4): 703-705 \\ DOI: https://doi.org/10.5114/ada.2021.108895
}

Food-dependent, exercise-induced anaphylaxis (FDEIA) is a distinct form of food allergy associated with anaphylaxis, in which clinical symptoms are elicited only when two factors coexist - intake of sensitizing food with subsequent physical exertion. It was first described by Maulitz et al. in 1979 [1]. The pathomechanism of FDEIA remains to be the subject of research, especially the relevance of physical exertion in the elicitation of IgEmediated systemic allergic reaction [2]. Most patients experience their symptoms after postprandial exercise (the typical sequence) [3]. Our team was the first who presented the case of FDEIA with atypical episodes when anaphylaxis occurred after exercise, followed by ingestion of the sensitizing allergen [4].

Nowadays, we present the second case of FDEIA with the reverse sequence of causative factors recognized in a birch pollen allergic patient after ingestion of soy milk. To discover the causative food allergen, we combined classic allergy testing with molecular allergy diagnostics.

A 41-year-old female patient with birch allergy experienced her first episode of anaphylaxis after intensive physical training and subsequent consumption of protein cocktail with the addition of soy milk. Pruritus, face and eyelids angioedema appeared about 10 min after the culprit food was ingested. Despite taking $10 \mathrm{mg}$ of levocetirizine, the patient developed generalized urticaria, abdominal pain, dyspnoea with wheezing and required Emergency Room treatment. The clinical history revealed that she had previously experienced oral allergy syndrome (OAS) after ingestion of carrot, avocado, pepper, tree nuts (hazelnuts, almonds and walnuts), Rosaceae fruit: apples, raspberries, blackberries, cherries and soy milk. There were also episodes of abdominal cramping pain caused by soy milk. Oropharyngeal symptoms occurred when the patient consumed a small amount of soy milk just before combining it with a protein shake.
To evaluate the triggering allergen, skin prick tests (SPT) with a standard set of aeroallergens and food allergens (Allergopharma, Reinbeck, Germany) were performed. The positive result was obtained to birch, hazel, linden-tree, dust mites, mugwort. Specific IgE antibodies (Euroimmun Medizinische Labordiagnostika, Lübeck, Germany) were detected against birch and other Fagales trees (alder, hazel, oak), Dermatophagoides pteronyssinus and Dermatophagoides farinae, egg white, hazelnut, apple, but not for soybean. For OAS and anaphylaxis-related foods (avocado, pepper, apple, raspberries, potato, carrot, egg, soy milk, protein cocktail), prick by prick tests were carried out accompanied by prick tests with fresh extracts of nuts (hazelnut, almond, walnut) and soybean, suspended in Coca solution without phenol (50\% of $0.9 \% \mathrm{NaCl}$ and $50 \%$ of glycerol) in concentration of $5 \%$ (vol/vol). Sensitization to soybean and soy milk was confirmed (wheal size: 15/8 mm, 8/8 mm). Skin testing was also positive to other tested allergens except for protein cocktail (without soy milk) and egg. The profile of sensitization based on component-resolved diagnosis was established (FABER, Centri Associati di Allergologia Molecolare, Rome, Italy) (Table 1). Increased concentration of specific IgE against molecules belonging to the PR-10-like family was detected but not against available recombinant allergens of soy and its extract. Bet $v 1$ homologue-soy allergen Gly $m 4$ was not obtainable in the multiplex-microarray assay.

The patient underwent provocation challenges with aspirin and exercise but no signs of anaphylaxis or changes in spirometric values were observed. A food challenge associated with exercise was not performed due to a high risk of a severe allergic reaction.

The soybean allergy is rare in adults [5] and so far, as a causative allergen in FDEIA, it has been reported three times [5-7]. Sensitization to allergens Gly $m 5$ and Gly $m 6$

Address for correspondence: Krzysztof Gomułka PhD, Department of Internal Medicine, Pneumonology and Allergology, Wroclaw Medical University, Wroclaw, Poland, phone: +48 7178426 47,

e-mail: kgomulka@wp.pl

Received: 25.07 .2020 , accepted: 14.08 .2020$. 
Table 1. Molecular sensitization profile; the range of serum specific lgE: negative $\leq 0.01 \mathrm{FIU} / \mathrm{ml}$; positive $\geq 0.30 \mathrm{FIU} / \mathrm{ml}$

\begin{tabular}{|c|c|c|c|}
\hline Allergen source & Allergen & $\begin{array}{l}\text { Type: M: allergenic molecules } \\
\text { E: allergen extract }\end{array}$ & Value \\
\hline Celery & Api g 1 & M & $8.16 \mathrm{FIU} / \mathrm{ml}$ \\
\hline Peanut & Ara h 8 & $M$ & $5.85 \mathrm{FIU} / \mathrm{ml}$ \\
\hline Birch & Bet v 1 (pollen) & $\mathrm{E}$ & 13.45 \\
\hline Birch & Bet $v 1$ & M & $17.81 \mathrm{FIU} / \mathrm{ml}$ \\
\hline Chestnut & Cas s (seed) & $\mathrm{E}$ & $1.96 \mathrm{FIU} / \mathrm{ml}$ \\
\hline Carob & Cer si (seed) & $\mathrm{E}$ & $1.18 \mathrm{FIU} / \mathrm{ml}$ \\
\hline Chickpea & Cic a & $\mathrm{E}$ & $0.78 \mathrm{FIU} / \mathrm{ml}$ \\
\hline Hazelnut & Cor a (pollen) & $E$ & $5.85 \mathrm{FIU} / \mathrm{ml}$ \\
\hline Hazelnut & Cor a 1 (pollen) & M & $8.54 \mathrm{FIU} / \mathrm{ml}$ \\
\hline Mites & Der $\mathrm{f} 1$ & M & $0.79 \mathrm{FIU} / \mathrm{ml}$ \\
\hline Mites & Der $\mathrm{f} 2$ & M & $0.79 \mathrm{FIU} / \mathrm{ml}$ \\
\hline Mites & Der $p$ & $\mathrm{E}$ & $1.5 \mathrm{FIU} / \mathrm{ml}$ \\
\hline Mites & Der $p 2$ & $M$ & $3.75 \mathrm{FIU} / \mathrm{ml}$ \\
\hline Walnut & Jug r (seed) & E & $0.78 \mathrm{FIU} / \mathrm{ml}$ \\
\hline Apple & Mal d 1 & M & $0.79 \mathrm{FIU} / \mathrm{ml}$ \\
\hline Soybean & Gly m & E & 0 \\
\hline Soybean & Gly $\mathrm{m} 1$ & M & 0 \\
\hline Soybean & Gly m Agglutinin & M & 0 \\
\hline Soybean & Gly m TI & $M$ & 0 \\
\hline
\end{tabular}

is particularly associated with a high risk of anaphylaxis $[6,7]$. Severe reactions to soy after an intake of unprocessed soy products (soy drinks) in patients sensitized to birch have also been reported $[6,8]$. It is the result of cross-reactivity of major birch pollen allergen Bet $\checkmark 1$ and its structural homologue Gly $m 4$ belonging to pathogenesis-related proteins (PR10) [8, 9]. In our case, FDEIA occurred in the context of soy milk ingestion. Such mildly processed soy products are likely to contain a high amount of native Gly $m 4$ [8] and because of its lability, this allergen molecule is in low abundance in commercial soybean extracts [9]. It could be the reason why extractbased sensitization tests with soybean were negative in our patient, while prick by prick tests with a fresh soy extract and soy milk gave a positive response. High serum Bet $v$ 1-specific IgE levels and positive results to the birch pollen-related fruits and vegetables indicate a soybean allergy due to Bet v 1 cross-reactions. However, Gly m 4 was not accessible in an extended panel of allergen molecules. IgE-sensitization to soybean (oropharyngeal symptoms, abdominal pain when soy milk consumed, positive prick by prick tests) is undoubted but the causative molecule was not possible to indicate. The results of CRD suggest more possibilities of potential episodes of FDEIA in the presence of physical exertion or other cofactors.
This case is also an atypical episode of FDEIA in which reversed clinical symptoms appeared. To our knowledge, this is the second report of this type. The first description concerned a patient with both classic FDEIA episodes and an unusual sequence [4]. In our patient, anaphylaxis was only associated with the inverted sequence but so far it was the only episode of systemic reaction. Unlike the previous case, the symptoms occurred after intense, short-term physical exertion. Accordingly, regardless of the stimulus sequence, physical effort leads to anaphylaxis. This is consistent with the alleged mechanism of FDEIA - increased intestinal permeability causes greater absorption of the allergen. The question remains why the reverse sequence in FDEIA is so rare. Our case confirms the need for further dissemination of knowledge about the reverse sequence of events in the course of FDEIA. Unawareness can lead to wrong diagnosis and erroneous recommendations. Patients with FDEIA should not consume causative food allergens not only $4 \mathrm{~h}$ before starting physical activity but also $2 \mathrm{~h}$ after its cessation. Another aim of this report is to show that in the case of FDEIA, successful diagnostics needs to combine allergy testing but above all require understanding of the disease nature.

\section{Conflict of interest}

The authors declare no conflict of interest. 


\section{References}

1. Maulitz RM, Pratt DS, Schocket AL. Exercise-induced anaphylactic reaction to shellfish. J Allergy Clin Immunol 1979; 63: 433-4.

2. Barg W, Mędrala W, Wolańczyk-Mędrala A. Exercise-induced anaphylaxis: an update on diagnosis and treatment. Curr Allergy Asthma Rep 2011; 11: 45-51.

3. Farrell A, Judge C, Redenbaugh V, et al. Food-dependent exercise-induced reactions: lessons from a 15-year retrospective study. Ir J Med Sci 2019; 188: 815-9.

4. Wolańczyk-Mędrala A, Barg W, Radlińska A, et al. Fooddependent exercise-induced anaphylaxis-sequence of causative factors might be reversed. Ann Agric Environ Med 2010; 17: 315-7.

5. Taramarcaz T, Hauser C, Eigemann PA. Soy anaphylaxis. Allergy 2001; 56: 792.

6. Adachi A, Horikawa T, Shimizu H, et al. Soybean b-conglycinin as the main allergen in a patient with food-dependent exercise-induced anaphylaxis by tofu: food processing alters pepsin resistance. Clin Exp Allergy 2009; 39: 167-73.

7. Yun J, Wienholt L, Loblay R. Food-dependent exercise-induced anaphylaxis (FDEIA) due to Gly $\mathrm{m} 6$ in soy. Intern Med J 2014; 44 (Suppl 4): P94.

8. Treudler R, Werner M, Thiery J, et al. High risk of immediatetype reactions to soy drinks in 50 patients with birch pollinosis. I Investig Allergol Clin Immunol 2008; 18: 482-95.

9. Mittag D, Vieths S, Vogel L, et al. Soybean allergy in patients allergic to birch pollen: clinical investigation and molecular characterization of allergens. J Allergy Clin Immunol 2004; 113: 148-54. 